\title{
Effects of a Relative-Frequency Elicitation Question on Likelihood Judgment Accuracy: The Case of External Correspondence
}

Paul C. Price, California State University, Fresno

In three experiments, college students judged the likelihood that they chose the correct alternative for each of 40 two-alterna-tive, generalknowledge items. They responded either to a rela-tive-frequency elicitation question ("Out of $\mathbf{1 0 0}$ questions for which you felt this certain of the answer, how many would you answer correctly?") or to a probability elicitation question ("What is the probability that you chose the correct answer?"). J udg-ments in response to the relative-frequency elicitation question tended to be lower, exhibit less scatter, and express complete certainty less often than judgments in response to the probability elicitation question. Two types of explanation for these effects are considered. First, the effect of the relative-frequency elicitation question may be to reduce random response error in participants' likelihood judgments. Second, the relativefrequency elicitation question may encourage the use of frequency information and simpler algorithms for making likelihood judgments.

Behavioral decision researchers have shown repeatedly that the way one person asks another for a judgment can have important effects on thejudgment itself. These effects are typically referred toas framing effects (Yates, 1990). The present research concerns one such framing effect in the domain of likelihood judgment. Specifically, it concerns the effects of a relative-frequency elicitation question (e.g., "In 100 cases like this one, how many times would target event

Thanks go to Frank Yates, Hal Arkes, Eric Stone, and Kevin Biolsi, whose ideas provided the impetus for this research.

Thanks also go to Colleen Doolin, Mary Lloyd, and Winston Sieck, who helped with the data collection and analysis. Address reprint requests to Paul C. Price, Department of Psychology, California State University, Fresno, 5310 North Campus Drive, Fresno, CA 93740-8019. E-mail: paulpri@csufresno.edu. 
E occur?") as opposed to the more typical probability elicitation question (e.g., "In this particular case, what is the probability that E will occur?"). What differences, if any, are there in people's likelihood judgments in response to the two types of elicitation question and which type, if either, elicits more accurate likelihood judgments? Obviously, the answers to these questions have practical implications for those who elicit or make likelihood judgments in important contexts like medicine, law, and business. The answers to these questions also have theoretical implications for those who wish to understand the nature of human likelihood judgment.

\section{INTE RNAL CONSISTENCY}

A number of studies have addressed the effects of a relative-frequency el icitation question on one aspect of likelihood judgment accuracy: internal consistency. Likelihood judgments are internally consistent to the extent that they conform to the rules of probability theory (Yates, 1982). The conjunction rule, for example, states that the probability of the conjunction of two events can be no greater than the probability of either of the two events taken alone. Consider, however, how people normally respond to the Tversky and K ahneman (1983) Linda problem, in which Linda is described as a 31-year-old woman who studied philosophy in college and is concerned with issues of social justice. Most people judge it to be more likely that Linda is a feminist bank teller than that Linda is a bank teller, which is impossible according to the conjunction rule. As Tversky and Kahneman first reported, however, the rate of conjunction errors on similar problems can be reduced substantially by reframing the problem in terms of relative frequencies. Gigerenzer (1994), for example, asked people to estimate, out of 100 women who fit Linda's description, the number who are bank tellers and thenumber whoarefeminist bank tellers. Thepercentage of people who made conjunction errors dropped from 88 to $16 \%$ (see also F iedler, 1988). It should be noted, however, that one replication of this procedure by J ones, J ones, and Frisch (1995) revealed a much smaller effect for the Linda problem and no effect at all for a similar problem.

Bayes's theorem is another rule of probability theory, which specifies how new information combines with the prior probability of an event to determine the posterior probability of that event. Consider, however, how people respond to the Kahneman and Tversky (1973) lawyer-engineer problem, in which a man named J ack is described as being conservative, having little interest in politics, and counting among his hobbies carpentry and solving mathematical puzzles. People are informed either that J ack has been chosen randomly from a pool of 70 engineers and 30 lawyers or that he has been chosen randomly from a pool of 30 engineers and 70 lawyers. Then they are asked to judge the probability that J ack is an engineer. Although Bayes's theorem states that the base rate of engineers in the pool must be taken into account-and precisely how it must be taken into account-people's judgments are affected hardly at all by this information. Again, however, people's likelihood judgments come closer to the normative standard when a relative-frequency el icitation question 
is used. When J ones et al. (1995) asked people how many of 100 men fitting J ack's description would be engineers (given an original pool of 1000 lawyers and engineers), more of them appeared to take the base rate into account. This same phenomenon has been reported and extensively analyzed by Gigerenzer (1991, 1994; Gigerenzer \& Hoffrage, 1995).

\section{EXTE RNAL CORRESPONDE NCE}

There is, then, some evidence that a relative-frequency question elicits likelihood judgments that have greater internal consistency than does a probability question. Until now, however, there has been almost no research on the effects of a relative-frequency elicitation question on a second aspect of likelihood judgment accuracy: external correspondence. External correspondence is the degree to which a person's likelihood judgments accord with the actual occurrence or nonoccurrence of the target events under consideration (Yates, 1982). The literature on overconfidence, for example, is concerned with one aspect of external correspondence (e.g., Adams \& Adams, 1961; Fischhoff, Slovic, \& Lichtenstein, 1977; Lichtenstein, Fischhoff, \& Phillips, 1982; Keren, 1991). Do people's judgments of the likelihood of some target event "match" the relative frequency with which the target event occurs? The answer to this question is often no; people tend to be overconfident.

In at least one study of over confidence, researchers havevaried the el icitation question. In a general-knowledgetask, Fischhoff et al. (1977) asked participants to judge the odds (in addition to the probability) that they had chosen the correct alternative for each of several two-choice, general-knowledge items. Making odds judgments, however, had no effect on the degree of overconfidence participants exhibited.

Despite this null result, there are at least three reasons that additional research on the effects of an alternative elicitation question (specifically, a relative-frequency elicitation question) on external correspondence is warranted. First, whereas the relative-frequency question has been hypothesized to be easier, or in some sense more natural, for people to understand (Gigerenzer, 1994), the odds question seems more difficult. People rarely think or communicate in terms of odds except in unusual contexts such as sports betting. For this reason, it seems possible that a relative-frequency question may have effects on external correspondence even though an odds question does not. Second, Fischhoff et al. (1977) were interested primarily in overconfidence and did not study the effects of their odds elicitation question on other aspects of external correspondence. As discussed presently, thereare many other interesting aspects of external correspondence that may or may not be affected by a relative-frequency elicitation question. A final reason to study the effects of a relative-frequency question on external correspondence is research showing that people performing a general-knowledge task often estimate the number or percentage of general-knowledge questions they answered correctly during the experiment with a high degree of accuracy (Gigerenzer, Hoffrage, \& Kleinbolting, 1991; Sniezek, Paese, \& Switzer, 1990). This suggests that people 
may haveaccess to accurate relative-frequency information to which a relativefrequency elicitation question may draw their attention. For all of these reasons, the present experiments were conducted to see how a relative-frequency elicitation question-analogous to those used in previous research on internal consistency-affects various aspects of external correspondence.

\section{GENERAL-KNOWLEDGE TASK}

For this initial study of the effects of a relative-frequency elicitation question on the external correspondence of people's likelihood judgments, a standard general-knowledge task was used (Fischhoff et al., 1977). Specifically, each participant responded to 40 two-alternative, general-knowledge items. For each item, the participant chose the alternative that he or she believed to be correct (each item included exactly one correct alternative) and then judged the likelihood that heor she had, in fact, chosen the correct alternative. The participant's likelihood judgments could range from 100, meaning he or she was certain of having chosen the correct alternative, down to 50, meaning the participant thought it as likely as not that he or she had chosen the correct answer. In the relative-frequency condition, the numerical response represented the number out of 100 similar questions the participant would expect to answer correctly. In the probability condition, the response represented the probability (expressed as a percentage) that he or she had chosen the correct alternative for that item.

This procedure is convenient for collecting a large number of likelihood judgments from each participant that can be analyzed in terms of their external correspondence. That is, each participants' likelihood judgments can be analyzed in terms of their correspondence with the actual correctness or incorrectness of his or her chosen alternatives. Furthermore, the external correspondence of likelihood judgments elicited by the relative-frequency question can be compared to the external correspondence of likelihood judgments elicited by the probability question.

\section{EXTERNAL CORRESPONDENCE: DECOMPOSITIONS OF THE MEAN PROBABILITY SCORE}

The data analytic approach used in the present research is a common one in the analysis of likelihood judgment accuracy (Winkler \& Murphy, 1968; Yates, 1994). First, each participant's mean probability score ( $\overline{\mathrm{PS}}$, also called the Brier Score; Brier, 1965) was computed according to the following formula:

$$
\overline{\mathrm{PS}}=\frac{1}{N} \sum_{i=1}^{N}\left(f_{i}-d_{i}\right)^{2} .
$$

In the present research, $\mathrm{N}$ is the number of general-knowledge items the participant responds to, $f_{i}$ is the participant's likelihood judgment for generalknowledge item $i$ expressed as a probability from 0 to 1 , and $d_{i}$ is the outcome 
index for general-knowledge item i. This outcome index takes on a value of 1 if the participant chose the correct alternative and a value of 0 if the participant chose the incorrect alternative for general-knowledge item $\mathrm{i}$. Thus, the mean probability score is the mean squared deviation of the participant's likelihood judgments from the actual outcomes and it constitutes a measure of the overall external correspondence of the participant's likelihood judgments, which can be compared across the two elicitation question conditions. N ote that the mean probability score can vary from 0 to 1 , with lower values indicating better external correspondence. A useful comparison value of the mean probability score is that obtained by a participant who expresses complete ignorance by making a judgment of 50 (which enters into the formula above as .5) for every general-knowledge item. This participant would achieve a mean probability score of .2500 .

To gain further insight into which components of external correspondence are affected by the relative-frequency elicitation question, each participant's mean probability score was decomposed in two different ways (see Yates, 1982, for details). The Murphy decomposition (Murphy, 1973) requires that the judgments be rounded to specific categories $\left(f_{j}=.5, .6, \ldots, 1.0\right)$ before calculating the two independent components of interest: the calibration index and the discrimination index. The calibration index is computed as

$$
\mathrm{Cl}=\sum \frac{\mathrm{Cl}_{\mathrm{j}}}{\mathrm{N}},
$$

where

$$
\mathrm{Cl}_{\mathrm{j}}=\mathrm{N}_{\mathrm{j}}\left(\mathrm{f}_{\mathrm{j}}-\overline{\mathrm{d}}_{\mathrm{j}}\right)^{2} .
$$

In the present research, $\mathrm{N}_{\mathrm{j}}$ is the number of likelihood judgments in category $\mathrm{j}$ and $\overline{\mathrm{d}}_{\mathrm{j}}$ is the proportion of items answered correctly given judgments in category $\mathrm{j}$. The calibration index is a measure of how closely a participant's likelihood judgments match the long-term relative-frequencies of the target event (i.e., answering a general-knowledge item correctly).

The discrimination index is computed as

$$
D I=\sum \frac{D_{j}}{N},
$$

where

$$
D I_{j}=N_{j}\left(\bar{d}_{j}-\bar{d}\right)^{2} .
$$

The only new term in this equation, $\bar{d}$, is the overall proportion of items answered correctly. The discrimination index is a measure of the extent to which the participant makes different judgments for (i.e., discriminates between) items he or she has answered correctly and items he or she has answered 
incorrectly. Note that because the calibration and discrimination indexes are independent of one another, either one of them may be affected positively or negatively by a relative-frequency elicitation question without implying anything about the other.

The Yates decomposition of the mean probability score produces three independent components of interest - the bias, slope, and scatter indexes-which provide a somewhat different perspective on external correspondence (Yates, 1994, 1982; Yates \& Curley, 1985). Bias, sometimes called calibration-in-thelarge, is the difference between the participant's mean likelihood judgment and percentage correct:

$$
\text { Bias }=\bar{f}-\bar{d} .
$$

It is often taken as a measure of the participant's over- or underconfidence, which is appropriate here because the target event is that "my chosen answer is correct." Slope is the difference between the participant's mean likelihood judgment when he or she chose the correct alternative and the participant's mean likelihood judgment when he or she chose the incorrect alternative:

$$
\text { Slope }=\bar{f}_{1}-\bar{f}_{0} .
$$

Like the discrimination index, it is a measure of the degree to which the participant distinguishes items he or she knows the answer to from items he or she does not know the answer to. Scatter is the weighted mean of two conditional variances: the variance of the participant's likelihood judgments when he or she chose the correct alternativeand thevariance of the participant's judgments when he or she chose the incorrect alternative:

$$
\text { Scatter }=\frac{\mathrm{N}_{1} \operatorname{Var}\left(\mathrm{f}_{1}\right)+\mathrm{N}_{0} \operatorname{Var}\left(\mathrm{f}_{0}\right)}{\mathrm{N}_{1}+\mathrm{N}_{0}} .
$$

Note that each variance is weighted by the number of judgments entering into it. Statistically, scatter is the variance in the participant's likel ihood judgments that is unrelated to whether or not he or she actually chose the correct alternative. Psychologically, scatter results from the participant's relying on weakly diagnostic or nondiagnostic cues in making his or her judgments or from applying his or her judgment strategy inconsistently. Again, because the bias, slope, and scatter indexes are independent of one another, any of them may be affected positively or negatively by a relative-frequency elicitation question without implying anything about the others. ${ }^{1}$

\footnotetext{
${ }^{1}$ In practice, however, slope and scatter tend to be correlated because they are both affected by the validity of the cues used by the judge (Yates \& Curley, 1985).
} 


\section{EXPERIMENTS la AND 1b}

Experiments $1 \mathrm{a}$ and $1 \mathrm{~b}$ constituted an initial attempt to see what effects, if any, a relative-frequency elicitation question has on the external correspondence of people's likelihood judgments. In both experiments, participants responded to 40 general-knowledge items and judged the likelihood, for each item, that they chose the correct alternative. In one condition, they responded to a relative-frequency elicitation question; in the other condition, they responded to a standard probability elicitation question.

It seemed possible that overconfidence, as indexed by the calibration index of the Murphy decomposition of the mean probability score and by the bias index of the Yates decomposition of the mean probability score would beaffected by the relative-frequency el icitation question. It is important to consider, however, that overconfidence depends to some extent on the difficulty of the generalknowledge items used. This is the so-called hard-easy effect (Lichtenstein \& Fischhoff, 1977). For difficult items, with correct response rates of $70 \%$ or less, overconfidence is generally observed. For easy items, with correct-response rates of $80 \%$ and up, no bias or even underconfidence is often observed. In other words, people's likelihood judgments are not sufficiently sensitive to changes in their accuracy. For this reason, Experiment la employed a set of difficult general-knowledge items expected to produce overconfidence, at least in the probability condition. Would there be less overconfidence in response to the relative-frequency elicitation question than in response to the probability elicitation question? Experiment $1 \mathrm{~b}$, on the other hand, employed a set of easier general-knowledge items expected to produce little if any overconfidence. If the relative-frequency elicitation question were to reduce overconfidence in Experiment 1a, what effect would it have in Experiment 1b? Would it have no effect, or would it lower bias, perhaps resulting in underconfidence? Of course, what about the effects of the relative-frequency elicitation question on other aspects of external correspondence?

\section{Method}

Participants. The participants were 127 undergraduate students at the University of Michigan. F ifty participated in Experiment la and 77 participated in Experiment 1b. They received partial credit in an introductory psychology course in return for their participation.

General knowledge items. Participants in Experiment la responded to a set of 40 two-alternative, general-knowledge items, which in extensive pilot research had produced correct-response rates between 60 and $70 \%$. This correct response rate has been shown repeatedly to result in overconfidence. Participants in Experiment $1 \mathrm{~b}$ responded to another set of 40 general-knowledge items, which included the 20 from Experiment la for which the correct-response rate was highest and 20 additional items written to produce a correct-response rate of about $80 \%$. This correct response rate has been associated with little 
or no overconfidence, and sometimes even underconfidence (e.g., Lichtenstein \& Fischhoff, 1977; Lichtenstein et al., 1982).

Procedure. Participants were tested in noninteracting groups of up to 15 individuals, with each one seated at a Macintosh II computer. Stimulus materials were presented, and participants' responses were recorded, by a program written in Hypercard. The experiment began with participants' reading a set of instructions that included a section encouraging partici pants to make accurate likelihood judgments, in which accuracy was described in terms of calibration. A bonus of $\$ 10.00$ was offered to the participants-one from each conditionwhose likelihood judgments were most accurate in this respect.

On each trial of the experiment, one general-knowledge item, along with two alternative answers, was presented on the monitor, along with instructions to "Please choose the answer you believe to be correct." Participants did so by using a mouse to point at and click on a pointing-finger icon next to their chosen answer. The icon became highlighted and remained so throughout the rest of the trial so that participants would remember which alternative they had chosen. Then the likelihood judgment elicitation question was presented. For participants who had been randomly assigned to the relative-frequency condition, it read as follows. "N ow, imagine 100 questions about which you felt as certain of the answer as you do of the answer to this one. How many of those 100 questions would you answer correctly? (Please enter a number from 50 to 100 .)" For participants who had been randomly assigned to the probability condition, it read as follows. "Now, what is the probability that you chose the correct answer? (Please enter a probability from $50 \%$ to $100 \%$.)" Upon typing an acceptable response (an integer from 50 to 100, and the return key, the screen was cleared and the next trial began.

\section{Results}

Experiment 1a. Table 1 shows, for each condition, the means and standard deviations of participants' percentages correct, mean likelihood judgments, mean probability scores, Murphy decomposition scores (i.e., calibration and discrimination indexes), and Yates decomposition scores (i.e., bias, slope, and scatter) under both conditions. Note first that neither participants' likelihood judgments, percentages correct, nor mean probability scores differed statistically significantly across conditions. ${ }^{2}$ Likewise, neither of the M urphy decomposition scores-the calibration index and the discrimination index-differed statistically significantly across conditions. As for the Yates decomposition scores, neither bias nor slope differed statistically significantly across conditions. Scatter, however, was statistically significantly lower (i.e., better) for participants who responded to the relative-frequency elicitation question than for participants who responded to the probability elicitation question.

Another interesting result was initially reveal ed by theaggregate calibration graph presented in Fig. 1. This graph shows participants' percentage correct

${ }^{2}$ All $p$ values reported are two-tailed. 
TABLE 1

Means and Standard Deviations of Percentage Correct, Mean Likelihood J udgment, and External Correspondence Scores by Elicitation Question Condition in

Experiments la and $\mathbf{~} \mathbf{l b}$

\begin{tabular}{|c|c|c|c|c|c|c|c|c|}
\hline \multirow[b]{2}{*}{ Condition } & \multicolumn{8}{|c|}{ Accuracy measure } \\
\hline & $\overline{\mathrm{d}}^{\mathrm{a}}$ & $\bar{f}^{b}$ & $\overline{\mathrm{PS}^{\mathrm{c}}}$ & $\mathrm{Cl}^{\mathrm{C}}$ & $\mathrm{DI}^{\mathrm{d}}$ & Bias $^{e}$ & Slope ${ }^{d}$ & Scatter $^{\mathrm{C}}$ \\
\hline \multicolumn{9}{|c|}{ Experiment 1a } \\
\hline \multicolumn{9}{|c|}{ Relative Frequency } \\
\hline$M$ & .6760 & .7225 & .2170 & .0377 & .0341 & .0465 & .0785 & $.0238 *$ \\
\hline SD & .0762 & .0733 & .0452 & .0277 & .0148 & .0798 & .0596 & .0092 \\
\hline \multicolumn{9}{|l|}{ Probability } \\
\hline M & .6640 & .7245 & .2330 & .0483 & .0320 & .0605 & .0723 & .0325 \\
\hline SD & .0820 & .0653 & .0487 & .0310 & .0219 & .0947 & .0764 & .0067 \\
\hline \multicolumn{9}{|c|}{ Experiment $1 b$} \\
\hline \multicolumn{9}{|c|}{ Relative Frequency } \\
\hline$M$ & .7682 & .7718 & .1785 & .0364 & .0278 & .0039 & .0951 & $.0303^{*}$ \\
\hline SD & .0778 & .0636 & .0342 & .0192 & .0179 & .0923 & .0595 & .0101 \\
\hline \multicolumn{9}{|l|}{ Probability } \\
\hline$M$ & .7767 & .7890 & .1796 & .0423 & .0290 & .0122 & .0940 & .0369 \\
\hline SD & .0803 & .0683 & .0412 & .0251 & .0184 & .0887 & .0588 & .0077 \\
\hline
\end{tabular}

a $\bar{d}$ is the mean percentage correct.

$\mathrm{b} \overline{\mathrm{f}}$ is the mean likelihood judgment expressed as a probability from 0 to 1 .

c Lower values indicate better external correspondence.

d Higher values indicate better external correspondence.

e Values closer to zero indicate better external correspondence.

* This mean under the relative-frequency condition is statistically significantly lower than the mean under the probability condition, $\mathrm{p}<.05$.

as a function of their likelihood judgments (rounded to the nearest decile), aggregated across participants, separately for each condition. The number nearest each point on the graph is the number of judgments represented by that point. Note that participants in the relative-frequency condition appear to have made fewer extreme judgments than participants in the probability condition. To examinethis result more closely, the number of times each participant made judgments of " 100 " and " 50 " (before rounding for analysis) were tallied. Whereas participants in the relative-frequency condition made a mean of 3.60 judgments of " 100 " (SD = 3.24), participants in the probability condition made a mean of 7.76 judgments of "100" ( $S D=4.61)$, $t(48)=3.69, p<.05$. Likewise, whereas participants in the relative-frequency condition made a mean of 8.08 judgments of " 50 " (SD $=7.71$ ), participants in the probability condition made a mean of 12.04 judgments of " 50 " (SD = 7.17). This difference was marginally statistically significant, $\mathrm{t}(48)=1.88, \mathrm{p}<.10$.

Experiment 1b. Table 1 also shows, for each condition in Experiment 1b, the means and standard deviations of participants' percentages correct, mean likelihood judgments, mean probability scores, and M urphy and Yates decomposition scores. Again, the scatter index from the Yates decomposition of the 


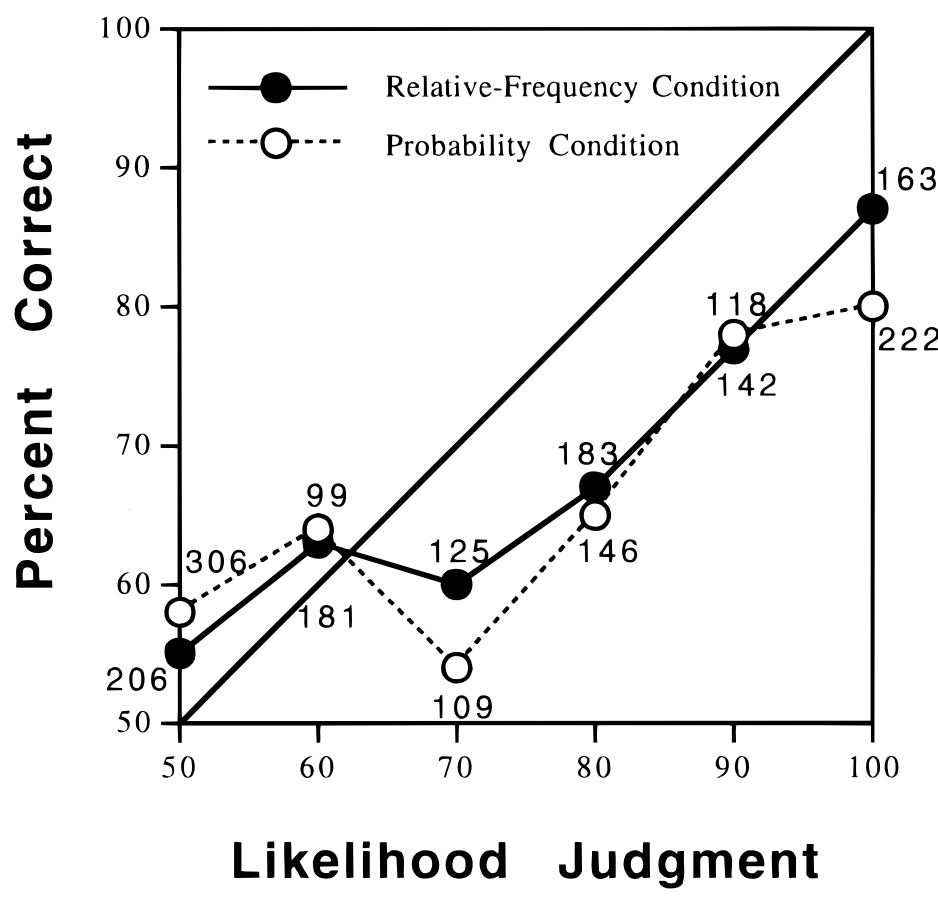

FIG. 1. Aggregate calibration graph for participants in relative-frequency condition and probability condition of Experiment la. The number nearest each point is the number of judgments represented by that point.

mean probability score was the only one of these dependent variables to differ statistically significantly across conditions; it was lower (i.e., better) in the relative-frequency condition than in the probability condition.

Also as in Experiment 1a, participants in the relative-frequency condition made fewer judgments of "100" than did participants in the probability condition. Whereas participants in the relative-frequency condition made a mean of 9.16 judgments of " 100 " (SD $=5.91)$, participants in the probability condition made a mean of 14.47 judgments of "100" ( $S D=5.79), t(48)=4.23, \mathrm{p}<.05$. They did not, however, make statistically significantly fewer judgments of " 50 ". Whereas participants in the relative-frequency condition made a mean of 7.00 judgments of " 50 " (SD $=6.39$ ), participants in the probability condition made a mean of 8.47 judgments of " 50 " (SD $=4.37$ ). This difference, however, was not statistically significant, $\mathrm{t}(48)=1.25, \mathrm{p}=.21$.

\section{Discussion}

The relative-frequency question elicited likelihood judgments that exhibited less scatter than did the standard probability question. This result might be explained by the additional fact that participants in the relative-frequency condition were less likely to express complete certainty, and to some extent complete uncertainty, about the correctness of the alternatives they chose than were participants in the probability condition. Moderating one's judgments of " 100 " and " 50 " will generally bring those judgments closer to the conditional 
mean likelihood judgment (i.e., the mean likelihood judgment conditioned on having chosen the correct alternative or on having chosen the incorrect alternative). This, in turn, will lower the conditional variance of the likelihood judgments and thereby lower the scatter index. Thus, these results are consistent with the hypothesis that the primary effect of a relative-frequency elicitation question, as compared to a probability elicitation question, is to reduce the tendency to express either complete certainty or complete uncertainty (i.e., reduce the tendency to make judgments of " 100 " and " 50 "). Experiment 2 was designed to test this hypothesis more directly.

\section{EXPERIMENT 2}

In essence, Experiment 2 was a within-subjects replication of Experiments $1 a$ and $1 b$. That is, participants responded to the same set of general-knowledge items twice, once responding to the probability elicitation question for each item and again responding to the relative-frequency elicitation question for each item. This design has several advantages over the between-subjects design of Experiments la and lb. First, the within-subjects design allows one to assess the existence of carryover effects. Does repeatedly responding to the relativefrequency question affect how people then respond to the probability question, or vice versa? This possibility is suggested by the work of Reeves and Lockhart (1993) on internal consistency. They found that the rate of conjunction errors was reduced when the target events (e.g., a student will be offered J ob A, J ob $B$, and both J obs A and B) were described by relative-frequency information (e.g., how many others have applied for those jobs) as opposed to case-specific information (e.g., characteristics of the student and of the jobs). Furthermore, the rate of conjunction errors on problems containing case-specific information was lower when participants had previously made judgments about one that contained relative-frequency information. It is possible, then, that when the relative-frequency elicitation question is presented first, its effects on external correspondence may carry over to the probability condition. Second, having participants make judgments in response to both elicitation questions puts them in a position to state their beliefs about the normative relationship between the two sets of judgments. Do they believe that their probability and relative-frequency judgments ought to be the same? Or do they believe that they need not necessarily be the same?

Perhaps most importantly, the within-subjects design allows us to see whether the effects of the relative-frequency elicitation question on external correspondence are due primarily to people's being less willing to express complete certainty and complete uncertainty in response to that question. Specifically, their external correspondence under the two conditions can be examined having eliminated from the data those items for which each participant re sponded with complete certainty or uncertainty in the probability condition but with less than complete certainty or uncertainty in the relative-frequency 
condition. If external correspondence is no longer better under the relativefrequency condition, then the effects were probably due to the set of items eliminated from the analysis.

\section{Method}

Participants. The participants were 68 undergraduate students at the University of Michigan. They participated in return for partial credit in an introductory psychology course.

Procedure. The 40 general-knowledge questions used in Experiment 2 were the same ones used in Experiment 1a. The procedure was essentially identical to that of Experiments $1 a$ and $1 b$, except that after responding to the 40 generalknowledge items under one condition, participants responded to those same 40 general-knowledge items (presented in a different random order) under the other condition. The order of the two conditions was counterbalanced across partici pants. After responding under the first condition, participants read additional instructions that emphasized they would now respond to the same set of general-knowledge items, this time making their likelihood judgments in response to a different elicitation question.

After responding to all 40 general-knowledge items under the second condition, participants were presented with the following question.

To recap, you just judged the likelihood that you answered each of several general-knowledge questions correctly, once by responding to the question

"What is the probability that you answered this question correctly?" and another time by responding to the question

"Out of 100 questions for which you felt this certain, how many would you answer correctly?" What does your intuition tell you about the appropriate relationship between your judgments in response to these two questions? Please click on one of the two responses below and note that neither answer is necessarily correct.

Bel ow were the two alternatives, between which participants chose by pointing at and clicking on one or the other. The two alternatives read as follows.

(1) For a given general-knowledge question, the two judgments should be the same (e.g., if one responds " $75 \%$ " to the first question, then one should respond " 75 out of 100 " to the second).

(2) For a given general-knowledge question, the two judgments should not necessarily be the same (e.g., if one responds " $75 \%$ " to the first question, one should not necessarily respond "75 out of 100 " to the second).

When participants responded to this question, the experiment was over.

\section{Results}

Complete data set. Table 2 shows the means and standard deviations of partici pants' percentages correct, mean likeli hood judgments, mean probability scores, and Murphy and Yates decomposition scores for the relative-frequency and probability conditions. The statistical analysis of each of these dependent variables was a repeated-measures analysis of variance with condition as a within-subjects factor and order of conditions as a between-subjects factor. 
TABLE 2

Means and Standard Deviations of Percentage Correct, Mean Likelihood J udgment, and External Correspondence Scores by Elicitation Question Condition, for Complete and Abridged Data Sets in Experiment 2

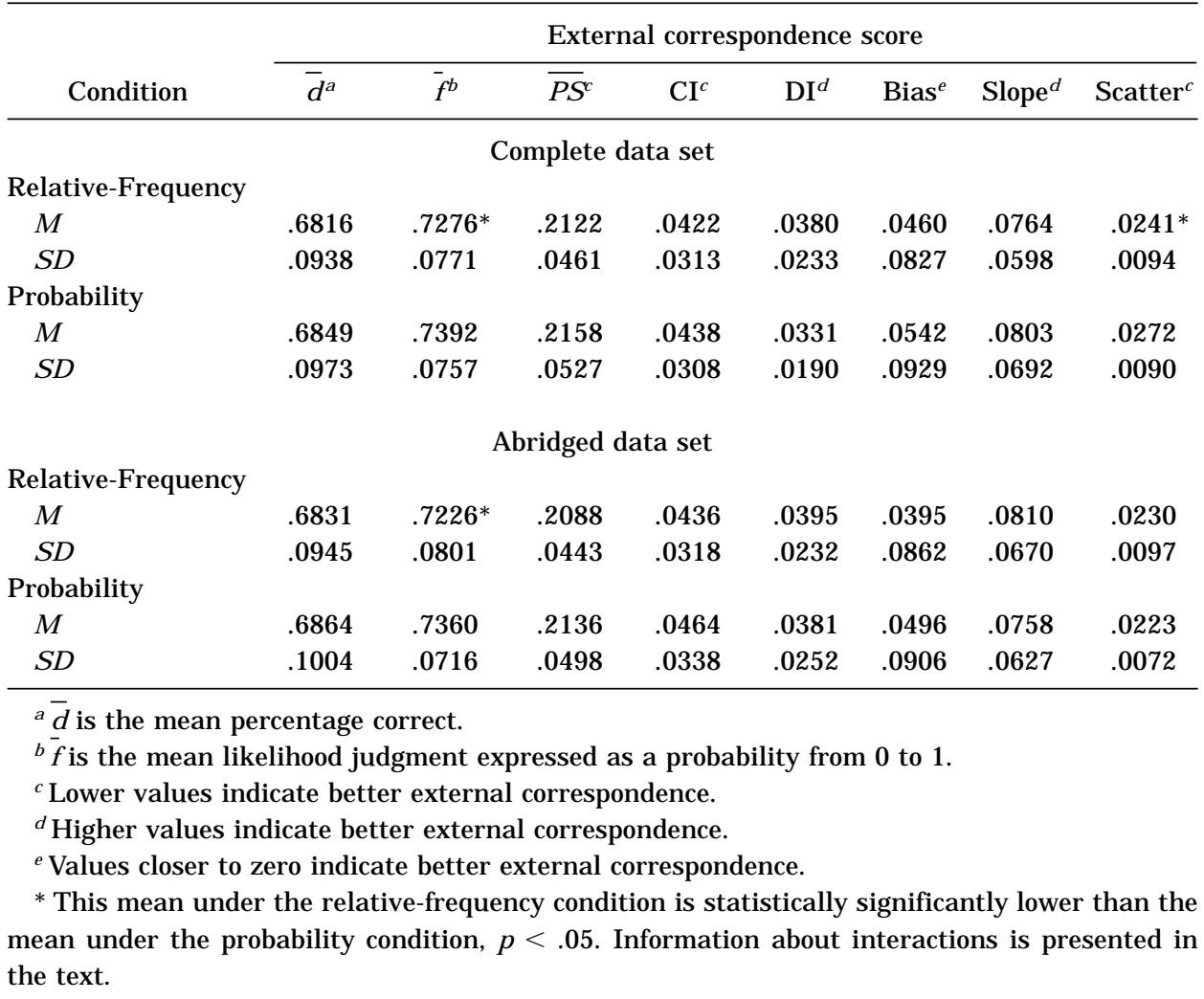

There were no statistically significant effects on the percentage correct or on the mean probability score. Unlike in Experiments $1 a$ and $1 b$, however, there was a statistically significant main effect on the mean likelihood judgment, $F(1,66)=5.68, p<.05$. Participants in the relative-frequency condition had a somewhat lower overall mean. There was also a statistically significant condition-by-order interaction effect on the mean likelihood judgment, $\mathrm{F}(1,66)=4.56, \mathrm{p}<.05$. When the probability condition preceded the relativefrequency condition, the effect on the mean likelihood judgment was larger than when the relative-frequency condition preceded the probability condition. Specifically, when the probability condition preceded the relative-frequency condition, the mean was 7189 in the relative-frequency condition (SD $=.0858$ ) and .7408 in the probability condition (SD $=.0831$ ). This difference was statistically significant by a dependent means t test, $t(33)=3.32, p<.05$. When the relative-frequency condition preceded the probability condition, however, the mean was .7363 in the relative-frequency condition $(S D=.0663)$ and .7375 in the probability condition (SD $=.0678$ ). This difference was not statistically significant, $\mathrm{t}(33)=0.07, \mathrm{p}>.05$. 
There were no statistically significant effects on either the calibration index or the discrimination index and none on either bias or slope. As in Experiments $1 a$ and $1 b$, however, there was a statistically significant main effect of condition on the scatter index, $F(1,66)=9.73, p<.05$. It was lower in the relativefrequency condition than in the probability condition. In addition, there was a marginally significant condition-by-order interaction effect on scatter, $F(1$, $66)=3.98, p=.05$. When the probability condition preceded the relativefrequency condition, the effect on the scatter index was larger than when the relative-frequency condition preceded the probability condition. Specifically, when the probability condition preceded the relative-frequency condition, the mean was .0232 in the relative-frequency condition $(S D=.0099)$ and .0283 in the probability condition (SD $=.0108$ ). This difference was statistically significant, $\mathrm{t}(33)=2.82, \mathrm{p}<.05$. When the relative-frequency condition preceded the probability condition; however, the mean was .0250 in the relativefrequency condition (SD $=.0084$ ) and .0261 in the probability condition $(S D=.0118)$. This difference was not statistically significant, $t(33)=1.35$, $\mathrm{p}>.05$.

Again, as in Experiments $1 a$ and $1 b$, participants expressed complete certainty less often in response to the relative-frequency elicitation question than in response to the probability el icitation question. Repeated measures analyses of variance, as described above, showed that participants made statistically significantly fewer judgments of "100" in the relative-frequency condition $(M=5.17, S D=5.52)$ than in the probability condition $(M=7.13, S D=6.53)$, $F(1,66)=11.30, p<.05$. They did not, however, makestatistically significantly fewer judgments of " 50 " in the relative-frequency condition ( $M=7.21, \mathrm{SD}=$ $6.68)$ than in the probability condition $(M=7.59, S D=5.74), F(1,66)=0.68$, $\mathrm{p}=.41$. There were no statistically significant interaction effects on either of these variables, although in both cases the effect was somewhat stronger when the probability condition preceded the relative frequency condition.

Abridged data set. Recall that a major goal of Experiment 2 was to determine to what extent the effects of the relative-frequency elicitation question on external correspondence were due to participants' responding to that question with fewer likel ihood judgments of 100 and 50. For this reason, the results of Experiment 2 were reanalyzed, eliminating all items for which participants made a judgment of 100 or 50 in the probability condition but made a less extreme judgment in the relative-frequency condition. If the effects of the relative-frequency question on external correspondence are eliminated too, then it can be concluded that these effects are due primarily to participants' moderating their most extreme likelihood judgments.

Table 2 also shows the means and standard deviations of participants' percentages correct, mean likelihood judgments, mean probability scores, and Murphy and Yates decomposition scores for each condition, based on the abridged data set. Note first of all that the statistically significant effect on the scatter index was now eliminated, suggesting that the reduction in scatter 
came about by participants' moderating their most extreme likelihood judgments. However, the statistically significant effect of elicitation question on the mean likelihood judgment remained, $F(1,66)=12.16, p<.05$, as did the statistically significant condition-by-order interaction effect, $F(1,66)=5.25$, $p<.05$. When the probability condition preceded the relative-frequency condition, the mean was somewhat lower in the relative-frequency condition (.7106, $\mathrm{SD}=.0871)$ than in the probability condition $(.7329, \mathrm{SD}=.0794)$. This difference was statistically significant, $\mathrm{t}(33)=4.33, \mathrm{p}<.05$. When the relativefrequency condition preceded the probability condition, however, the mean was about the same in the relative-frequency condition $(.7346, S D=.0732)$ as in the probability condition $(.7392, \mathrm{SD}=.0637)$. This difference was not statistically significant, $t(33)=0.80, p>.05$. Note that both the main effect of condition and the condition-by-order interaction effect on the mean likelihood judgment were somewhat stronger in the abridged data set than in the complete data set. Therefore, the tendency for participants to make less extreme likelihood judgments in response to the relative-frequency elicitation question does not appear to account for the entire pattern of results.

Perceived relationship between judgment types. Finally, recall that participants were asked whether they believed that their relative-frequency judgments ought to be the same as their probability judgments or whether it would be acceptable for them to differ. Interestingly, participants' responses to this question depended heavily on the order in which they were exposed to the two elicitation questions. Of the 34 participants who responded first to the probability elicitation question and then to the relative-frequency elicitation question, only 11 (32\%) thought that judgments in response to the two elicitation questions ought to be the same. Of the 33 participants who responded first to the relative-frequency question and then to the probability question (one failed to respond to this question), 25 (76\%) thought that judgments in response to the two elicitation questions ought to be the same. This difference is unlikely to have arisen if the response to this question were independent of the order of conditions, $\chi^{2}(\mathrm{~N}=67)=12.69, \mathrm{p}<.05$.

\section{DISCUSSION}

The results of Experiment 2 confirmed those of Experiments $1 \mathrm{a}$ and $1 \mathrm{~b}$. The relative-frequency question elicited likelihood judgments that exhibited less scatter. In addition, participants in Experiment 2 made statistically significantly lower mean likelihood judgments under the relative-frequency condition than under the probability condition. Perhaps the reason that this result was obtained in Experiment 2, but not in Experiments $1 \mathrm{a}$ and $1 \mathrm{~b}$, is that the withinsubjects design of Experiment 2 heightened the contrast between the two types of elicitation question. Experiment 2 also replicated the tendency for participants to express complete certainty less often in response to the relativefrequency question. There was some evidence that the effect on scatter was mediated by this tendency to make less extreme judgments in response to the 
relative-frequency elicitation question. The statistically significant difference between the mean scatter index under the two conditions, for example, was eliminated when the analysis excluded items to which participants responded with judgments of "100" or " 50 " in the probability condition but with less extreme judgments in the relative-frequency condition. On the other hand, the effect on the mean likelihood judgment actually became stronger in the abridged data set. For this reason, the tendency to make fewer extreme judgments cannot account for all effects of the relative-frequency elicitation question.

There was one other particularly interesting result of Experiment 2. People were far from unanimous in their beliefs about the normative relationship between their probability and relative-frequency judgments. Only slightly more than half reported that they thought likelihood judgments in response to the two el icitation questions ought to bethe same. In addition, people's beliefs about the relationship between their probability and relative-frequency judgments depended on the order in which they had been exposed to the two types of elicitation question. This result is consistent with the modest evidence for order effects on the mean likelihood judgment and on scatter from Experiment 2. Here is one possible integration of these results. When participants respond to the probability elicitation question first, they make their probability judgments in the usual manner. The relative-frequency elicitation question, then, causes them to change the way they make their judgments (see the General Discussion for some possibilities), thereby affecting their external correspondence. On the other hand, when participants respond to the relative-frequency elicitation question first, they begin by making their judgments in the alternative manner. The probability elicitation question, then, does not cause them to revert back to their usual manner of making their judgments (see Reeves \& Lockhart, 1993, for a similar result). F urthermore, participants who responded first to the probability elicitation question are aware of the fact that they have begun responding differently to the relative-frequency elicitation question. When asked whether judgments in response to the two elicitation questions ought to be the same, then, they either infer on the basis of their own behavior that they need not or they answer that they need not to justify their own behavior. Participants who responded first to the relative-frequency elicitation question, however, did not change the way they responded. For this reason, they infer on the basis of their own behavior that their judgments in response to the two elicitation questions ought to be the same or, again, they answer that they ought to be the same to justify their own behavior.

\section{GENERAL DISCUSSION}

The present experiments, results, and analyses make clear that the external correspondence of people's likelihood judgments is affected by a relativefrequency el icitation question as opposed to the standard probability el icitation question. In all three experiments, scatter was statistically significantly lower under the relative-frequency condition than under the probability condition. 
It should also be noted here that a number of other measures-the mean likelihood judgment, mean probability score, calibration index, and bias index-also tended to differ (in the direction of better external correspondence) under the relative-frequency condition across the three experiments. Figure 2 shows the effect size (i.e., the difference between the means under the two conditions divided by the pooled standard deviation; Rosenthal, 1991) for percentage correct, mean likelihood judgment, and the external correspondence scores for Experiments $1 \mathrm{a}, 1 \mathrm{~b}$, and 2 when the probability condition preceded the relative-frequency condition. Note the similarity of the entire pattern of results across experiments. In fact, a simple meta-analysis confirmed that the combined effect of the elicitation question was statistically significant for the mean likelihood judgment $(p=.006)$, the mean probability score $(p=.004)$, the calibration index $(p=.01)$, and of course the scatter index $(p<.0001)$. It was marginally statistically significant for the bias index $(p=.07) .^{3}$

The results of the three experiments also agreed in that participants under the relative-frequency condition tended to make judgments expressing complete certainty less often than did participants under the probability condition. Participants under the relative-frequency condition also tended to make judgments expressing complete uncertainty less often, but this effect was statistically significant only in Experiment 1a. Although making less extreme likelihood judgments can lead to the pattern of external correspondence effects described above, it appears that matters are not so simple. Again, when items for which participants made judgments of " 100 " or " 50 " in the probability condition but not in the relative-frequency condition were el iminated from the analyses, the effect on scatter was reduced, but the effect on the mean likelihood judgment actually became larger. This indicates clearly that the effects of the relative-frequency elicitation question are not limited to the most extreme judgments.

A more complete explanation for the present results might be provided by a recently developed class of models that incorporates random error into the likelihood judgment process (Erev, Wallsten, \& Budescu, 1994; J uslin, Olsson, \& Bjorkman, 1997; Pfeiffer, 1994; Soll, 1996). A key assumption of these models is that an objectivelikel ihood judgment is a function of (1) a subjectivelikelihood judgment and (2) random response error. Applying this class of model to the present results, one might hypothesize that the effect of the relative-frequency question is to reduce the amount of random response error in participants' likelihood judgments, perhaps because the meaning of the relative-frequency question is clearer and participants respond to it more consistently. If so, one might expect a reduction in random response error (depending on the precise distribution of the error term) to result in less extreme likelihood judgments (and therefore less bias), better calibration, and less scatter (see, e.g., J uslin

\footnotetext{
${ }^{3}$ The meta-analytic method used - which is called the Stouffer method by Mosteller and Bush (1954) - involves transforming the one-tailed $p$ values to $z$ scores, combining these (three) $z$ scores into a single $z$ score, and transforming the result back to a $p$ value (Rosenthal, 1991, pp. 85-86).
} 


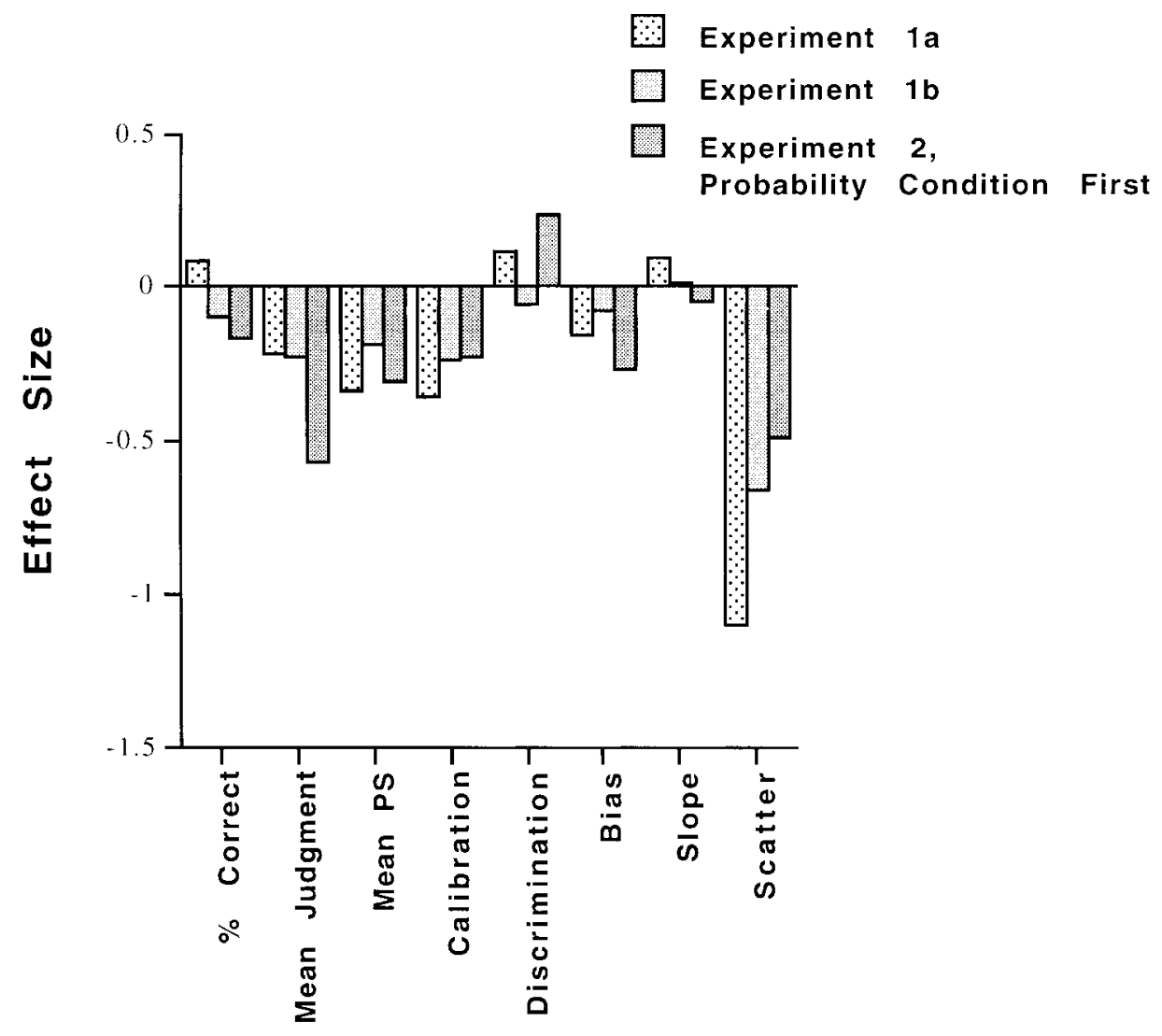

\section{Performance Measure}

FIG. 2. Effect sizes for all performance measures for Experiments $1 a, 1 b$, and 2 (when the probability condition preceded the relative-frequency condition). The effect size is the difference between the means under the two conditions divided by the pooled standard deviation.

et al., 1997, for details). This seems like an extremely promising avenue for future empirical research and model testing.

Note that this kind of explanation seems very different from the kind of explanation typically offered for the beneficial effect of relative-frequency questions and presentation formats on performance on tests of internal consistency. (Brase, Cosmides, \& Tooby, 1998; Cosmides \& Tooby, 1996; Gigerenzer, 1991; Gigerenzer \& H offrage, 1995; J ones et al., 1995; Tversky \& Kahneman, 1983). For example, some researchers have argued that the human cognitive system (and that of nonhumans as well) has evolved special algorithms for computing likelihoods based on data that take the form of event frequencies (Brase et al., 1998; Cosmides \& Tooby, 1996; Gigerenzer \& H offrage, 1995). As a result, these algorithms work well when the data take the form of event frequencies and work less well when the data take other forms (i.e., proportions or single-event probabilities). In addition, when the relevant data take the form of event frequencies, normative algorithms are often much simpler than they would be otherwise (Gigerenzer \& H offrage, 1995; Kleiter, 1994). 
It is possible, however, to apply this perspective to the present study too. For example, one can consider the overconfidence phenomenon in almanac question studies as a form of base rate neglect. Despite the fact that people know the fairly low relative frequency with which they are choosing the correct alternative (Gigerenzer, 1991; Sniezek et al., 1990), they continue to express high confidence that they have chosen the correct alternative on an item-byitem basis. The information they possess about the base rate with which they answer such questions correctly (i.e., their prior probability of answering a question correctly) seems to have little impact on their likelihood judgments in response to particular items. People's likel ihood judgments should improve, however, if they can be induced to begin with their prior probability and update it on the basis of item-specific information according to Bayes's theorem. What item-specificinformation? One possibility is their level of confidenceafter choosing an alternative and evaluating their choice in the usual manner. In terms of single-event probabilities or proportions, this general strategy suggests the following rather complex Bayesian algorithm:

$\mathrm{P}($ Correct $\mid \mathrm{C})=\frac{\mathrm{P}(\mathrm{C} \mid \text { Correct }) \mathrm{P}(\text { Correct })}{\mathrm{P}(\mathrm{C} \mid \text { Correct }) \mathrm{P}(\text { Correct })+\mathrm{P}(\mathrm{C} \mid \text { Incorrect }) \mathrm{P}(\text { Incorrect })}$.

In terms of frequencies, however, this general strategy suggests a much simpler algorithm,

$$
P(\text { Correct | C })=\frac{F(C \& \text { Correct })}{F(C)} \text {, }
$$

where $F$ is theabsolute frequency with which an event (or conjunction of events in this case) occurred (see also Gigerenzer \& Hoffrage, 1995). To the extent that the relative-frequency question encourages or allows people to use stored frequencies as their data and to use simpler al gorithms for making their likelihood judgments, those judgments ought to be better. In fact, it should be noted here that the relative-frequency elicitation question ("Out of 100 questions for which you felt this confident, how many would you answer correctly?") is a fairly direct request to use the simpler frequency-based algorithm above to judge the posterior probability $\mathrm{P}$ (Correct $\mathrm{C}$ ) . The extent to which this perspective-in addition to the perspective provided by the error models-can account for the present results is an important subject for future investigation. Perhaps they can even be combined, with the use of frequency data and simpler algorithms explaining the reduction in random response error under the relativefrequency condition. 


\section{REFERENCES}

Adams, J. K., \& Adams, P. A. (1961). Realism of confidence judgments. Psychological Review, 68, 33-45.

Brase, G. L., Cosmides, L., \& Tooby, J . (1998). Individuation, counting, and statistical inference: The role of frequency and whole-object representations in judgment under uncertainty. J ournal of Experimental Psychology: General, 127, 3-21.

Cosmides, L., \& Tooby, J . (1996). Are humans good intuitive statisticians after all? Rethinking some conclusions of the literature on judgment under uncertainty. Cognition, 58, 1-73.

Erev, I., Wallsten, T. S., \& Budescu, D. V. (1994). Simultaneous over- and underconfidence: The role of error in judgment processes. Psychological Review, 101, 519-527.

Fiedler, K. (1988). The dependence of the conjunction fallacy on subtlelinguistic factors. Psychol ogical Research, 50, 123-129.

Fischhoff, B., Slovic, P., \& Lichtenstein, S. (1977). Knowing with certainty: The appropriateness of extreme confidence. J ournal of Experimental Psychol ogy: Human Perception and Performance, 3, 552-564.

Gigerenzer, G. (1991). How to make cognitive illusions disappear: Beyond "heuristics and biases." European Review of Social Psychology, 2, 83-115.

Gigerenzer, G. (1994). Why the distinction between single-event probabilities and frequencies is important for psychology (and vice versa). In G. Wright and P. Ayton (Eds.), Subjectiveprobability (pp. 129-161). New York: Wiley.

Gigerenzer, G., \& Hoffrage, U. (1995). How to improve Bayesian reasoning without instruction: Frequency formats. Psychological Review, 102, 684-704.

Gigerenzer, G., H offrage, U., \& Kleinbolting, H. (1991). Probabilisticmental models: A Brunswikian theory of confidence. Psychological Review, 98, 506-528.

J ones, S. K., J ones, K. T., \& Frisch, D. (1995). Biases of probability assessment: A comparison of frequency and single-case judgments. Organizational Behavior and Human Decision Processes, 61, 109-122.

J uslin, P. (1994). The overconfidence phenomenon as a consequence of informal experimenterguided selection of almanac items. Organizational Behavior and Human Decision Processes, 57, 226-246.

J uslin, P., Olsson, H., \& Bjorkman, M. (1997). Brunswikian and Thurstonian origins of bias in probability assessment: On the interpretation of stochastic components of judgment. J ournal of Behavioral Decision Making, 10, 189-209.

Kahneman, D., \& Tversky, A. (1973). On the psychology of prediction. Psychological Review, 80, 237-251.

Keren, G. (1991). Calibration and probability judgments: Conceptual and methodological issues. Acta Psychologica, 77, 217-273.

Kleiter, G. (1994). Natural sampling: Rationality without base rates. In G. H. Fischer \& D. Laming (Eds.), Contributions to mathematical psychol ogy, psychometrics, and methodol ogy (pp. 375-388). New York: Springer-Verlag.

Lichtenstein, S., \& Fischhoff, B. (1977). Do those who know more also know more about how much they know? The calibration of probability judgments. Organizational Behavior and Human Performance, 20, 159- 183.

Lichtenstein, S., Fischhoff, B., \& Phillips, L. D. (1982). Calibration of probabilities: The state of the art to 1980. In D. Kahneman, P. Slovic, \& A. Tversky (Eds.), J udgment under uncertainty: Heuristics and biases (pp. 306-334). New York: Cambridge.

Mosteller, F. M., \& Bush, R. R. (1954). Selected quantitative techniques. In G. Lindzey (Ed.), Handbook of social psychology: Theory and method (Vol. 1, pp. 289-334). Cambridge, MA: Addison-Wesley. 
Murphy, A. H. (1973). A new vector partition of theprobability score. J ournal of Applied Meteorol ogy, 12, 595-600.

Pfeiffer, P. E. (1994). Are we overconfident in the belief that probability forecasters are overconfident? Organizational Behavior and Human Decision Processes, 40, 193-218.

Reeves, T., \& Lockhart, R. (1993). Distributional versus singular approaches to probability and errors in probabilistic reasoning. J ournal of Experimental Psychology: General, 122, 207-226.

Rosenthal, R. (1991). Meta-analytic procedures for social research. Newbury Park, CA: Sage.

Sniezek, J . A., Paese, P. W., \& Switzer, F. S. (1990). The effect of choosing on confidence in choice. Organizational Behavior and Human Decision Processes, 46, 264- 282.

Soll, P. J . (1996). Determinants of overconfidence and miscalibration: The roles of random error and ecological structure. Organizational Behavior and Human Decision Processes, 65, 117- 137.

Tversky, A., \& Kahneman, D. (1983). Extensional versus intuitive reasoning: The conjunction fallacy in probability judgment. Psychological Review, 90, 293-315.

Winkler, R. L., \& Murphy, A. H. (1968). “Good” probability assessors. J ournal of Applied Meteorol ogy, 7, 751-758.

Yates, J . F. (1982). External correspondence: Decompositions of the mean probability score. Organizational Behavior and Human Performance, 30, 132-156.

Yates, J . F. (1990). J udgment and decision making. New York: Prentice-Hall.

Yates, J . F. (1994). Subjective probability accuracy analysis. In G. Wright and P. Ayton (Eds.), Subjective probability. New York: Wiley.

Yates, J. F., \& Curley, S. P. (1985). Conditional distribution analyses of probabilistic forecasts. J ournal of Forecasting, 4, 61-73. 